\title{
Vimentin activation in early apoptotic cancer cells errands survival pathways during DNA damage inducer CPT treatment in colon carcinoma model
}

\author{
Souneek Chakraborty ${ }^{1,2}$, Aviral Kumar ${ }^{3}$, Mir Mohd Faheem², Archana Katoch ${ }^{1,2}$, Anmol Kumar $^{3}$, Vijay Lakshmi Jamwal ${ }^{4}$, \\ Debasis Nayak ${ }^{1,2}$, Aparna Golani ${ }^{3}$, Reyaz Ur Rasool ${ }^{1,2}$, Syed Mudabir Ahmad ${ }^{1,2}$, Jedy Jose ${ }^{3}$, Rakesh Kumar $\mathbb{0}^{5}$, \\ Sumit G Gandhi ${ }^{4}$, Lekha Dinesh Kumar ${ }^{3}$ and Anindya Goswami ${ }^{1,2}$
}

\begin{abstract}
Epithelial to mesenchymal transitions (EMT) is a preparatory process for cancer cells to attain motility and further metastasis to distant sites. Majority of DNA damaging drugs have shown to develop EMT as one of the major mechanisms to attain drug resistance. Here we sought to understand the resistance/survival instincts of cancer cells during initial phase of drug treatment. We provide a tangible evidence of stimulation of EMT factors in Apc knockout colorectal carcinoma model. Our results implied that CPT-treated Apc knockout cohorts depicted increased proinvasive and pro-survival factors (Vimentin/ $\mathrm{p}^{\mathrm{ser} 38}$ Vimentin \& NFkB). Moreover, by cell sorting experiment, we have observed the expression of Vimentin in early apoptotic cells (AnnexinV positive) from 36 to $48 \mathrm{~h}$ of CPT treatment. We also observed the expression of chimeric Sec-AnnexinV-mvenus protein in migrated cells on transwell membrane recapitulating signatures of early apoptosis. Notably, induction of Vimentin-mediated signaling (by CPT) delayed apoptosis progression in cells conferring survival responses by modulating the promoter activity of NFkB. Furthermore, our results unveiled a novel link between Vimentin and ATM signaling, orchestrated via binding interaction between Vimentin and ATM kinase. Finally, we observed a significant alteration of crypt-villus morphology upon combination of DIM (EMT inhibitor) with CPT nullified the background EMT signals thus improving the efficacy of the DNA damaging agent. Thus, our findings revealed a resistance strategy of cancer cells within a very initial period of drug treatment by activating EMT program, which hinders the cancer cells to achieve later phases of apoptosis thus increasing the chances of early migration.
\end{abstract}

\section{Introduction}

Epithelial to mesenchymal transition (EMT) is one of the earliest events in cancer cell metastasis. EMT can be

\footnotetext{
Correspondence: Lekha Dinesh Kumar (lekha@ccmb.res.in) or

Anindya Goswami (agoswami@iiim.ac.in)

${ }^{1}$ Academy of Scientific \& Innovative Research (AcSIR), CSIR-Indian Institute of Integrative Medicine, Jammu 180001, India

${ }^{2}$ Cancer Pharmacology Division, CSIR-Indian Institute of Integrative Medicine, Jammu 180001, India

Full list of author information is available at the end of the article.

Edited by S. Tait
}

viewed as a preparatory mechanism where the cells acquire the mesenchymal phenotype, by undergoing cytoskeletal rearrangements, to gain motility for further metastasis ${ }^{1}$. Intermediate filaments (IF) play a vital role during EMT progression by maintaining cellular stiffness $^{2}$. Out of the six major IFs, Vimentin (type 3 IFs) is considered as the most important facilitator for mesenchymal cellular stiffness. Vimentin gets expressed in epithelial cells only when EMT is activated otherwise they solitary express keratin as a major IF. Apart from its

\section{(c) The Author(s) 2019}

(c) (i) Open Access This article is licensed under a Creative Commons Attribution 4.0 International License, which permits use, sharing, adaptation, distribution and reproduction in any medium or format, as long as you give appropriate credit to the original author(s) and the source, provide a link to the Creative Commons license, and indicate if changes were made. The images or other third party material in this article are included in the article's Creative Commons license, unless indicated otherwise in a credit line to the material. If material is not included in the article's Creative Commons license and your intended use is not permitted by statutory regulation or exceeds the permitted use, you will need to obtain permission directly from the copyright holder. To view a copy of this license, visit http://creativecommons.org/licenses/by/4.0/. 
cytoskeletal role, phosphorylated counterpart of Vimentin acts as signaling agent during EMT and interacts with numerous proteins to execute strong cellular survival responses $^{3}$.

Although EMT is reported to contribute towards drug resistance $^{4,5}$, the mechanism by which EMT facilitates cancer cells to acquire resistance lacks adequate research. Whether EMT is a collateral event during drug resistance or a programmed strategy for the cancer cells to escape the drug-induced stresses, is an emerging field of study. Majority of drug development programs in cancer aim at selective elimination of cancer cells by modulating apoptotic mechanisms like DNA damage, oxidative stress, etc. Families of drugs which damage DNA and hinder its repair mechanisms are widely used in cancer therapeutics, with majority of FDA-approved drugs belonging to this category ${ }^{6-8}$. But from the very beginning, these class of drugs confront the problem of resistance ${ }^{4,9-13}$. For that reason, there is an urgent need to investigate the cause of failure of this class of DNA damaging drugs.

In this research, we provide strong evidences of activation of EMT and survival factors during initial phases of treatment with DNA damaging agent Camptothecin in Apc floxed colorectal carcinoma model as well as in colorectal/lung carcinoma cell lines. Notably, we found the expression of Vimentin that coexists along with early apoptotic population further hinders apoptotic progression unless otherwise accelerated in presence of EMT inhibitor (Di-indoyl Methane). Additionally, we have described the role of ATM kinase in Vimentin phosphorylation thus modulating its pro-survival and promigratory function.

\section{Results}

\section{CPT treatment confers Vimentin activation and EMT} induction in colon carcinoma

The typical role of EMT in drug resistance and stemness acquisition in cancer cells is recently understood but little is known about how cancer cells survive by activating EMT with an aim to circumvent apoptosis/anoikis ${ }^{5}$. In our preliminary studies, distinct morphological changes of epithelial cells were observed when treated with DNA damaging agents (data not shown). Rationally, we sought to examine the effects of CPT-mediated DNA damages on EMT activation in cancer cells. Western blot analysis showed a gradual increase in the expression of EMT specific marker-Vimentin, in three different epithelial cell lines (HCT-116, Sw-620, and A549) when treated with increasing concentrations of CPT (ranging from 50 to $250 \mathrm{nM}$ ) for $36 \mathrm{~h}$ (Supplementary Fig. 1A). Similar results were obtained following treatment with increasing concentrations of 5-Flurouracil (5-FU), Doxorubicin, and Cisplatin (Supplementary Fig. 1B and 2). Since $250 \mathrm{nM} \mathrm{CPT}$ is optimal for apoptotic induction ${ }^{14}$ and given the fact that
Vimentin was adequately expressed at this concentration, $250 \mathrm{nM}$ CPT was employed for further time-dependent studies. When HCT-116 and A549 cells were subjected to $250 \mathrm{nM}$ CPT (0-48 h), diminishing E-cadherin expression followed by gradual up-regulation of Vimentin levels achieved indicating the induction of EMT in these cells (Fig. 1a). Although, Vimentin phosphorylation is an important event for both EMT persistence and cell survival $^{3}$, CPT-treated cells exhibited a steep increase in $\mathrm{p}^{\text {ser38}}$ Vimentin expression up to $36 \mathrm{~h}$, then dropped sharply afterwards (Fig. 1a). While a steady amplification of Snail-1, ATM, and $\beta$-catenin expression were obtained by CPT treatment (12-36 h), however, ATM and Snail-1 expression diminished at $48 \mathrm{~h}$ (Fig. 1a). The immunocytochemistry results validated our immunoblots experiments. The localization of Vimentin in the nucleus, cytosol, and in migrating structures (indicated by red arrow heads) additionally confirmed its role in survival responses $^{15}$. Conversely, the gradual disappearance of E-cadherin from the cellular surface was also noticed (Fig. 1b). The bright field microscopy revealed that the epithelial morphology of vehicle-treated HCT-116 cells disappeared within $24 \mathrm{~h}$ of $\mathrm{CPT}$ treatment and majority of cells attained mesenchymal morphology at $36 \& 48 \mathrm{~h}$ (Fig. 1c). Interestingly, the apoptotic population was predominantly noted at $48 \mathrm{~h}$ (blue arrow head for apoptotic bodies and red arrow head for mesenchymal cells). To reason the cellular protrusion like structures in Fig. 1b (red arrows), we evaluated the invasive capability of the CPT-treated cells by FITC-gelatin degradation assay revealing a significant increase in gelatin degradation following CPT treatment, implying the acquisition of invasiveness by HCT-116 / A549 cells (Fig. 1d, Supplementary Fig. 1C).

$A p c$ conditional knock out murine colorectal carcinoma model was used to evaluate EMT initiation by CPTinduced DNA damage in vivo. This model mimics early tumor development along the entire length of the small intestine $^{16,17}$. Mice cohorts were treated with three different concentrations of CPT $(0.4,0.8$, and $1.2 \mathrm{mg} / \mathrm{kg})$ for 24 and $48 \mathrm{~h}$ immediately after induction. An early appearance of "crypt progenitor" phenotype in the CPTtreated mice was found $(0.4 \mathrm{mg} / \mathrm{kg})$ compared to vehicle. However, at higher concentration of CPT $(1.2 \mathrm{mg} / \mathrm{kg})$, the villus and crypt boundaries started to restore (Supplementary Fig. 3A). The immunohistochemistry analyses of intestinal sections obtained from CPT-treated mice $(0.4 \mathrm{mg} / \mathrm{kg})$ revealed a significant rise in Vimentin and $\mathrm{p}^{\mathrm{ser} 38}$ Vimentin expression compared to control cohorts manifesting EMT induction in vivo (Fig. 1e, Supplementary Fig. 3B). At higher CPT concentrations (0.8 and $1.2 \mathrm{mg} / \mathrm{kg}$ ) Vimentin expression was more as compared to vehicle but gradually diminished as compared to the $0.4 \mathrm{mg} / \mathrm{kg}$ CPT-treated mice. (Fig. 1e, f). But the 


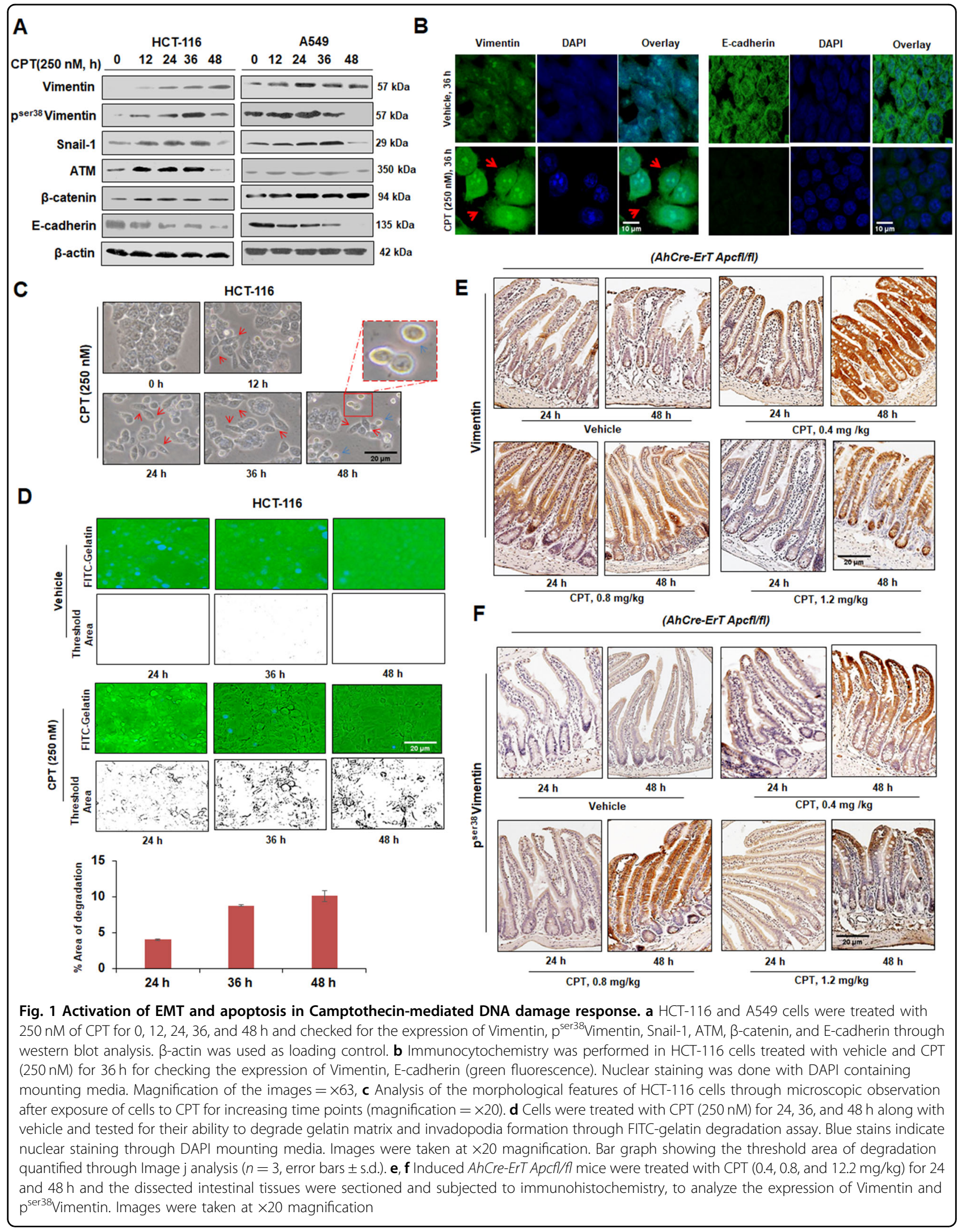


expression of $\mathrm{p}^{\mathrm{ser} 38}$ Vimentin was elevated in both 0.4 \& $0.8 \mathrm{mg} / \mathrm{kg}$ concentrations at $48 \mathrm{~h}$, which eventually decelerated following $1.2 \mathrm{mg} / \mathrm{kg}$ of CPT treatment (Fig. 1f, Supplementary Fig. 3C). Notably, the curing dose of CPT $(1.2 \mathrm{mg} / \mathrm{kg})$ was unable to completely nullify the associated EMT which might hamper apoptosis. These results clearly suggest that the tendency of DNA damage induced by chemotherapeutic agents accelerates EMT and prometastatic signaling which might facilitate the escape of the cancer cells during early stages of chemotherapeutic regime resulting in chemo-resistant secondary tumors ${ }^{18}$.

\section{Simultaneous occurrence of EMT and apoptosis in CPT- treated cells}

Apoptosis involves multistep processes and each step encompasses unique cytological and molecular signatures. While flipping of phosphatidyl serine (PS) to outer membrane leaflets is one of the early events in apoptosis $^{19-21}$, damage of chromatin reticulum or DNA fragmentation occurs at later stages ${ }^{22}$.To examine whether any apoptotic parameters prevailed in the CPT-treated cells, HCT-116 and A549 cells were treated with CPT (0-48 h) followed by AnnexinV-PI staining. FACS analysis data clearly revealed that with the increase in the time of CPT treatment there was a gradual decrease in the percentage of viable cells with simultaneous increase in the percentage of early apoptotic cells. Interestingly, early apoptotic population was almost equivalent to the viable population at $36 \mathrm{~h}$ of $\mathrm{CPT}$ treatment $(\sim 47 \%=$ viable $=$ early apoptotic population) in HCT-116 cells. Similarly, CPT-treated A549 cells showed equivalent percentages of viable and early apoptotic fractions at $36 \mathrm{~h}$, indicating that EMT induction goes hand in hand with apoptotic stimulation upon CPT treatment (Fig. 2a, Supplementary Fig. 4).

Since initial disruption of mitochondrial transmembrane potential $(\Delta \Psi \mathrm{m})$ occurs quite early in apoptotic cascades $^{19}$, we sought to evaluate whether there is any loss of $\triangle \Psi \mathrm{m}$ upon CPT exposure in a time-dependent manner. TMRE fluorescence quantification by FACS depicted a gradual increase in the low fluorescent TMRE fraction of cells (M1 quadrant), indicating periodical loss of $\Delta \Psi \mathrm{m}$ upon CPT treatment (Fig. $2 \mathrm{~b}$ ). $\mathrm{Bcl}_{2}$ family proteins play a vital role in cytochrome $\mathrm{c}$ release by disrupting the transmembrane of mitochondria. The western blotting analysis unearthed significant augmentation of proapoptotic markers - Bax and truncated Bid, with downmodulation of pro-survival protein $-\mathrm{Bcl}_{2}$ in presence of CPT (Fig. 2c). The in vivo data also revealed an inverse relationship between $\mathrm{Bax}$ and $\mathrm{Bcl}_{2}$ expression in agreement with our in vitro results (Fig. 2d). As early stages of apoptosis are quite flexible and a lot of survival proteins can manipulate these stages $^{20,23}$, we investigated more committed stages of apoptosis in time-dependent CPT treatment. Since, prominent cleavage of Caspase-3 and PARP-1 was observed at $48 \mathrm{~h}$ of CPT treatment (Fig. 2e), this time point $(48 \mathrm{~h})$ could be considered as a junction where the cells fully commit to the apoptotic fate. To extensively investigate the relation between EMT and apoptosis, AnnexinV-FITC and propidium iodide (PI) stained HCT-116 cells were sorted into early apoptotic and viable fractions using MoFlo cell sorter. The immunoblot analysis of the early apoptotic fraction revealed an increased Vimentin expression $12 \mathrm{~h}$ onwards, whereas the viable cells expressed Vimentin only at $12 \mathrm{~h}$ of CPT treatment (Fig. 2f). The presence of Vimentin in early apoptotic cells validated co-existence of apoptosis and EMT in cells threatened by a DNA damaging agent. We sought to understand the same phenomena by transfecting CPT-treated HCT-116 cells with N3-secAnnexinV$m$ Venus construct ${ }^{24}$ developed specifically to tag apoptotic cells with flipped PS. Interestingly, cells expressing AnnexinV-mVenus (green fluorescence bordering the cells) depicted mesenchymal morphology in the bright field images (red arrows; Fig. 2g), underscoring coexistence of mesenchymal and apoptotic cells. The bright fluorescent cells (yellow arrows) were observed majorly at $48 \mathrm{~h}$ seemed to be a conglomeration of apoptotic bodies (Fig. 2g) ${ }^{21}$. Further, non-transfected CPTtreated HCT-116 cells $(12-36 \mathrm{~h})$ were supplemented with the conditional media from N3-secAnnexin $V-m V e n u s$ transfected cells. Accordingly, the conditional media containing secretory AnnexinV-mVenus chimeric protein interacted with the flipped Phosphatidylserine of nontransfected cells contemplating the green fluorescence validating our hypothesis (Supplementary Fig. 5). Interestingly, the transfected N3-secAnnexinV-mVenus cells traversed the Boyden chamber membrane confirming those apoptotic cells (due to CPT treatment) possessed enhanced migratory behavior (Fig. 2h) whereas vehicle (DMSO) treated chambers were devoid of any migratory cells (Fig. 2h). The cell scattering results also supported similar observations (Supplementary Fig. 6). Collectively, these above results demonstrate that during initial phases of drug treatment both early apoptotic as well as EMT phenotypes co-exist together within the same population of cells.

\section{EMT activation triggered pro-proliferative responses during CPT treatment in vitro and in vivo}

Oncogenic EMT not only acts as a preparatory mechanism for the cells to gain motility for further metastasis but also allows them to evade cell death by initiating survival signaling ${ }^{25,26}$. To study the effects of DNA damage on cell migration, Boyden chamber assay was performed where CPT-treated HCT-116 and A549 cells robustly traversed the membrane (Fig. 3a). The 3D spheroids assay with HCT-116 cells showed distinct 


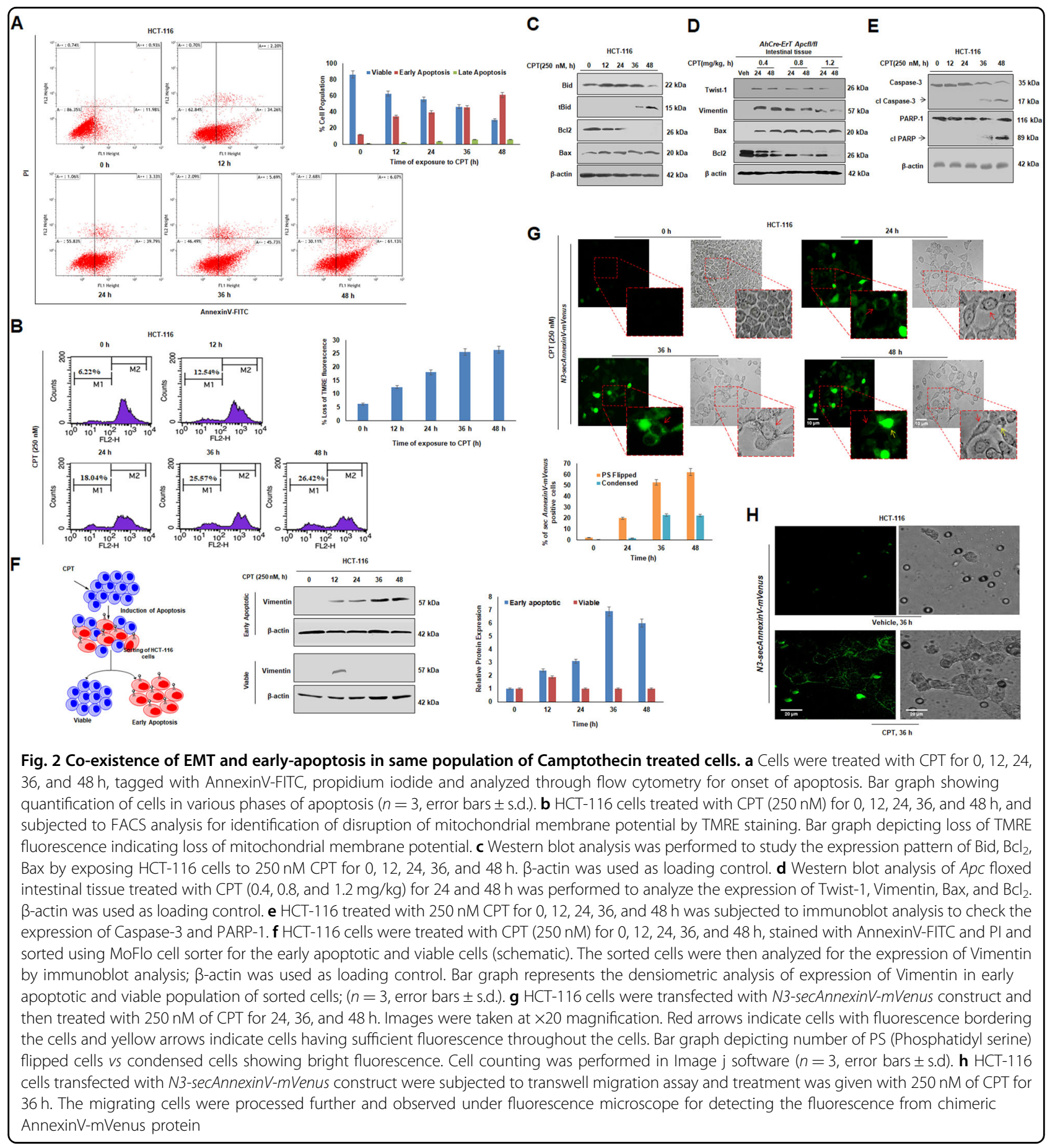

dispersion of cells from the spheroid periphery into the surrounding Matrigel upon treatment with CPT $(24 \mathrm{~h} /$ $36 \mathrm{~h} / 48 \mathrm{~h}$ ) as compared to control (Fig. 3b). Moreover, the cells displayed the maximum migration at $36 \mathrm{~h}$ as evident by the highest concentric radius (Red line- migratory front; Blue line- inner spheroid core). Further, a steady augmentation of MMP-2 and MMP-9 expression in HCT-116 cells was noticed following CPT treatment accompanied with increased gelatin degradation (by MMP2 and MMP-9) in time-dependent manner (Fig. 3c, d).

Next, we sought to evaluate whether there were any concurrent perturbations in cellular pro-survival signals due to CPT treatment. The western blot results clearly depicted an escalated $\mathrm{cFLIP}_{\mathrm{L} / \mathrm{S}}$ expression up to $24 \mathrm{~h}$ following a sharp attenuation at $48 \mathrm{~h}$ in presence of CPT, while Survivin showed a biphasic expression pattern 


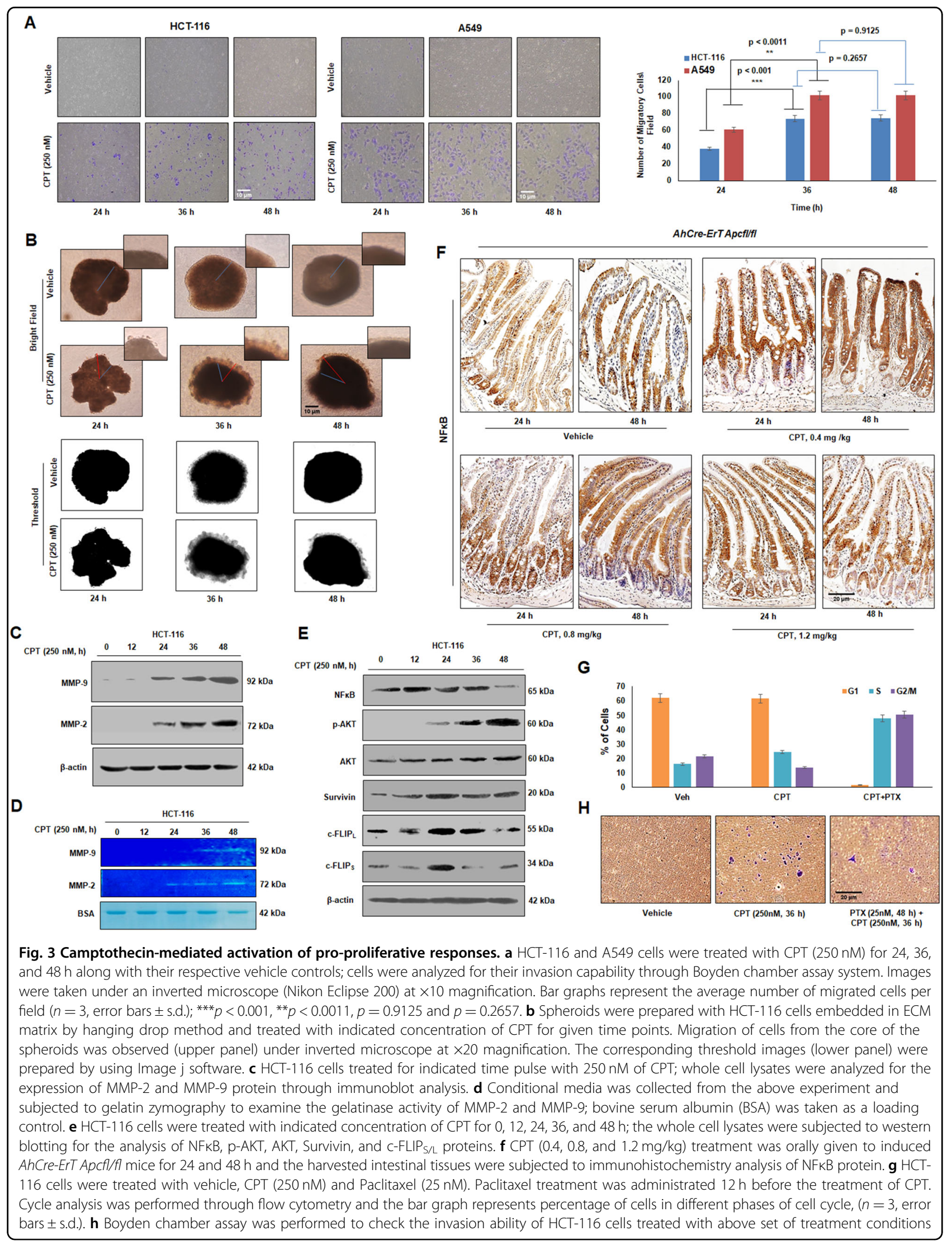


(Fig. 3e). Given that NFKB is a master regulator of cell survival responses ${ }^{27,28}$, its expression amplified at $12 \mathrm{~h}$ and the level was maintained up to $36 \mathrm{~h}$ followed by a steep down-modulation at $48 \mathrm{~h}$ (Fig. 3e). The immunohistochemistry analysis of CPT-treated Apc floxed mice unveiled high NFkB expression in $0.4 \mathrm{mg} / \mathrm{kg}$ CPT-treated cohorts compared to their respective vehicle controls at 24 and $48 \mathrm{~h}$ (Fig. 3f). The NFKB expression decreased significantly in $1.2 \mathrm{mg} / \mathrm{kg}$ CPT-treated cohorts compared to $0.4 \& 0.8 \mathrm{mg} / \mathrm{kg}$ (Fig. 3f, Supplementary Fig. 7).

Both EMT and apoptosis has been linked with cell cycle phases and cells in $G_{1}$ arrest showed preference towards migration and survivability compared to $\mathrm{G}_{2} \mathrm{M}$ arrested cells where apoptosis is favored ${ }^{29}$. In the current situation, when cells were blocked in $\mathrm{G}_{2} \mathrm{M}$ phase by paclitaxel $(25 \mathrm{nM})$ along with CPT, a significant inhibition in cell migration was observed along with diminished Vimentin expression (Fig. 3g, h and Supplementary Fig. 8). All these results collectively demonstrate a profound activation of survival responses due to the stimulation of EMT cascades.

\section{Induction of Vimentin confers apoptotic resistance and promotes survival responses due to CPT treatment}

In order to understand the function of Vimentin in modulating apoptosis, Di-indolyl methane (DIM)- a potent EMT blocker ${ }^{30-32}$ was used to nullify the CPTinduced EMT. Interestingly, EMT-blockade by DIM shifted the PARP-1 cleavage pattern to 24 and $36 \mathrm{~h}$ compared to CPT (without DIM) treatment where prominent PARP-1 cleavage was obtained only at $48 \mathrm{~h}$ (Fig. 4a). This shift in the PARP-1 cleavage pattern could be attributed to the decelerated Vimentin levels in presence of DIM thereby promoting cell death. The significance of Vimentin induction as an anti-apoptotic factor was further confirmed in CPT-treated HCT-116 cells, wherein, transient knock-down of Vimentin resulted in enhanced PARP-1 cleavage at $24 \mathrm{~h}$ in comparison to scramble transfected cells (Fig. 4b). Further, we observed the attenuation of survival proteins viz. cFLIP and NFKB in CPT-treated cells compared to scrambled controls wherein both cFLIP and NFKB expressions elevated gradually. Since, cFLIP possesses specific role in blocking FAS trafficking, which is one of the major mediators of apoptosis, the cells were subjected to Vimentin si-RNA knockdown followed by ICC analysis. Vimentin knockdown resulted in loss of cFLIP expression leading to significant FAS trafficking to the membrane (Fig. 4c). Additionally, Vimentin knockdown alleviated the invasive capability of CPT-treated HCT-116 cells as observed in the FITC-gelatin degradation assay (Fig. 4d and Bar graph).To examine the consequences of AKT inhibition, a major upstream activator of Vimentin ${ }^{33}$, the cells were treated with AT-7867 (allosteric AKT inhibitor) leading to a significant down-modulation of $\mathrm{p}^{\text {Ser38 }}$ Vimentin, cFLIP and NFKB in CPT-treated lane along with AT7867 compared to lane treated with CPT only indicating a direct correlation between survival factors and Vimentin (Fig. 4e). Since, NFkB expression was downmodulated in CPT plus si-Vimentin condition, we hypothesized that Vimentin could have a role in controlling $\mathrm{NF}_{\mathrm{K} B}$ transcription at its promoter level. We transfected the HCT116 cells with $\mathrm{NF} \kappa \mathrm{B}$ luciferase reporter construct and analyzed NFKB transcriptional activity through a DualGlo Luciferase Assay System. The luciferase activity of each sample was normalized by the luciferase activity of the PGL2 vector. The CPT-treated cells demonstrated a significant increase in luciferase activity that attenuated significantly in si-Vimentin condition (around threefold) (Fig. 4f). Together, these results suggest that Vimentin induction has an integrative role in controlling residual survival responses and progression through apoptosis by directly or indirectly regulating the transcriptional activation of $\mathrm{NF}_{\kappa} \mathrm{B}$ protein.

\section{ATM kinase activation is essential to maintain Vimentin related endeavors during DNA damage condition}

Recent evidences suggest that several components of DNA damage and repair pathway activate EMT inducing factors $^{34,35}$. ATM kinase, a DNA damage sensor, reportedly phosphorylates and activates Snail-1, a major transcription factor controlling EMT associated genes ${ }^{34}$. We explored how ATM kinase plays a central role in orchestrating Vimentin during CPT treatment in HCT116 cells. We have already demonstrated that the expression of ATM kinase was well synchronized with the $\mathrm{p}^{\text {ser38 }}$ Vimentin as well as Snail-1 levels in both HCT-116 and A549 cells (Fig. 1a). Hence, we hypothesized that ATM kinase might play an active role in phosphorylating Vimentin, in DNA damage condition. Therefore, when HCT-116 cells were treated with KU60019, a potent ATM kinase inhibitor, the expression of $\mathrm{p}^{\text {ser38 }}$ Vimentin declined significantly in presence of CPT (Fig. 5a). However, following silencing of Vimentin by siRNA, we could not find any significant change in expression of ATM kinase compared to corresponding control (Fig. 5b). Further, the role of ATM kinase in phosphorylating Vimentin was analyzed by transiently overexpressing ATM kinase in HCT-116 cells with pcDNA3.1(+) Flag-His-ATM wt. We observed significant rise in the level of $\mathrm{p}^{\text {ser38 }}$ Vimentin expression in lane overexpressing ATM kinase treated with $250 \mathrm{nM} \mathrm{CPT}$ as compared to lane treated with CPT only (Fig. 5c). Seeing that AKT plays a vital role in phosphorylating Vimentin ${ }^{33}$, in Fig. 4e, we observed that the expression of $\mathrm{p}^{\text {ser38}}$ Vimentin was still in considerable quantity in condition where AKT was blocked by AT7867. This indicates that other kinases also play a role in phosphorylating Vimentin. Although, in Fig. 5a, c, we 


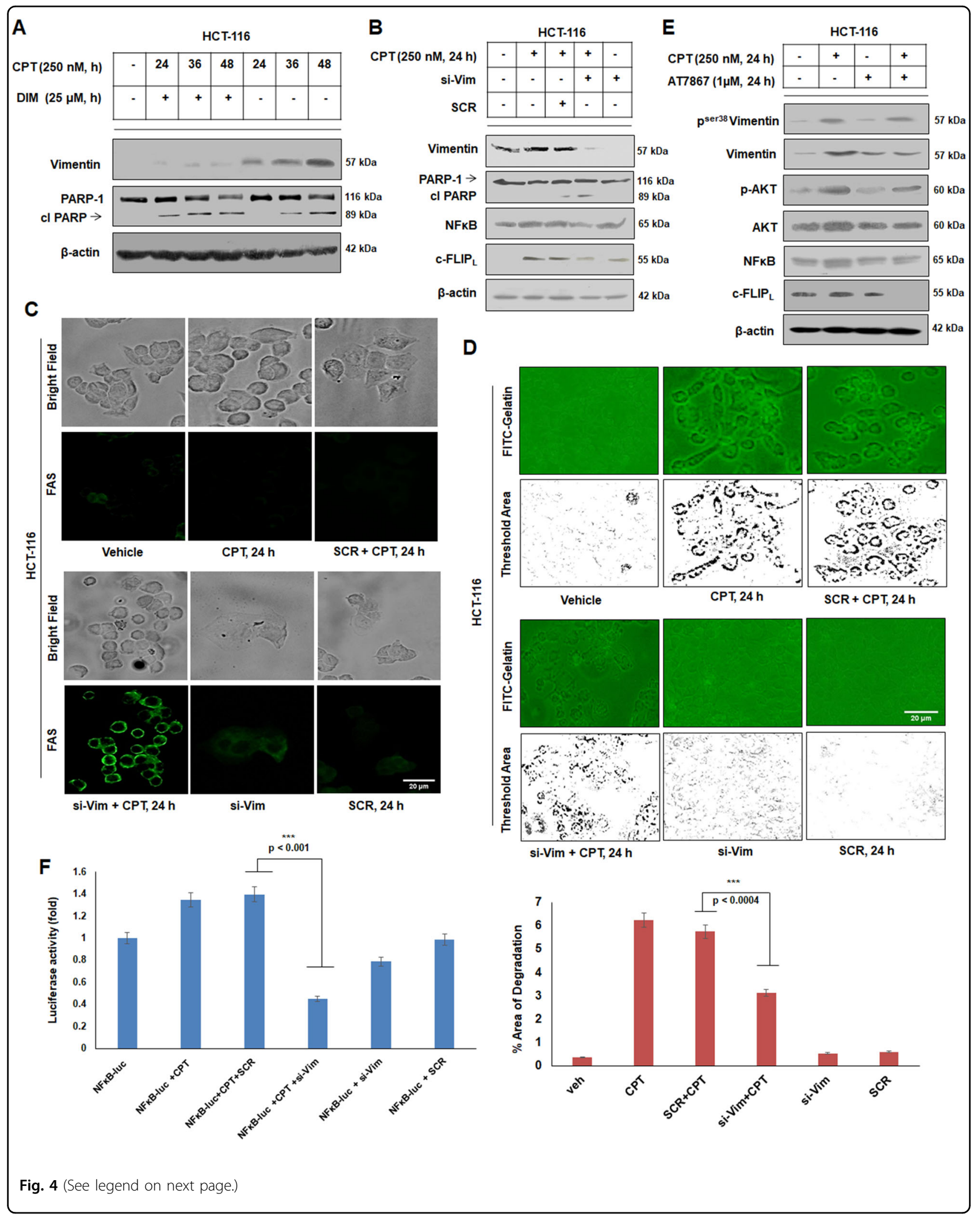




\begin{abstract}
(see figure on previous page)
Fig. 4 Induction of Vimentin hinders the progression through apoptosis. a HCT-116 cells were either treated with vehicle, CPT (250 nM) and CPT $(250 \mathrm{nM})+$ DIM $(25 \mu \mathrm{M})$ for indicated time points; whole cell lysates were prepared and subjected to western blot analysis of Vimentin and PARP-1 protein. b Cells were either transfected with vehicle, CPT (250 nM), SCR + CPT (250 nM), si-Vimentin plus CPT ( $250 \mathrm{nM}$ ) and si-Vimentin for 24 h; whole cell lysates were employed for western blot analysis of Vimentin, PARP-1, NFKB, and cFLIP. c Immunocytochemistry was performed for the indicated treatment conditions to examine the expression and trafficking of FAS ligand in HCT-116 cells. Images were taken by using Floid Cell Imaging Station; magnification $\times 20$. $\mathbf{d}$ Similar set of treatment conditions were used to check the cells capability for gelatin matrix degradation through FITC-gelatin degradation assay. Imaging was performed using Floid Cell Imaging Station at $\times 20$ magnification. The images were further analyzed and quantified for threshold area of degradation by Image $\mathrm{j}$ software and represented in bar graphs $\left(n=3\right.$, error bars \pm s.d.); ${ }^{* * *} p<0.0004$. e HCT-116 cells were either treated with vehicle, CPT (250 nM), AT7867 (1 $\mu \mathrm{M})$ and combination of both for $24 \mathrm{~h}$; whole cell lysates were subjected to western blot analysis for the expression of $\mathrm{p}^{\text {ser38}}$-Vimentin, Vimentin, p-AKT, AKT, NFKB, and CFLIP . f HCT-116 cells were either transfected with vector, NFKB-luc alone and/ or treated with NFkB-luc + CPT (250 nM), NFkB-luc + CPT (250 nM) + SCR, NFkB-luc + si-Vimentin + CPT (250 nM), NFkB-luc + si-Vimentin, and NFkBluc + SCR in 96 well plates for 24 h; luciferase activity was measured using Dual-Glo Luciferase Assay system (Promega). Normalization was done with luciferase activity of vector $\left(n=3\right.$, error bars \pm SD). ${ }^{* *} p<0.001$
\end{abstract}

found that ATM kinase aids in phosphorylating Vimentin at Serine 38 position, we now sought to understand the importance of ATM in Vimentin phosphorylation over AKT in CPT-mediated DNA damage condition. We, therefore, employed both AKT and ATM inhibitors (AT7867 \& KU60019) together as well as separately in CPTtreated conditions. Interestingly, we observed that the expression of $\mathrm{p}^{\mathrm{ser} 38}$ Vimentin diminished sharply in $\mathrm{KU} 60019+\mathrm{CPT}$ lane as compared to AT-7867 + CPT. Moreover, the expression of $\mathrm{p}^{\mathrm{ser} 38}$ Vimentin reduced more profoundly where both the kinases were blocked in presence of $250 \mathrm{nM} \mathrm{CPT} \mathrm{(Fig.} \mathrm{5d).} \mathrm{Additionally,} \mathrm{the} \mathrm{expres-}$ sion of NFKB also decreased upon blocking ATM/AKT separately as well as in the lane where both the kinases were blocked simultaneously as compared to CPT alone condition. Next, we sought to understand the integrative role of ATM in promoting the anti-apoptotic function of Vimentin. Intriguingly, by inhibiting ATM, cleaved caspase-3 augmented sharply in ectopic Vimentin lane, which otherwise depleted in GFP-Vimentin lane in presence of CPT (Fig. 5e). Subsequently, the implication of ATM in Vimentin-mediated migration of HCT-116 cells treated with CPT was examined and the results showed a significant rise in cell migration in Vimentin overexpressed condition. Further, ATM inhibition by KU60019 drastically affected the migration of cells (Fig. 5f, bar graph). Since, Vimentin in its phosphorylated form has been reported to interact with various kinases and based on our previous results (Fig. 5a, c), we speculated that Vimentin and ATM kinase could directly/ indirectly interact with each other. To examine our hypothesis, co-immunoprecipitation assay clearly unveiled the interaction between ATM and Vimentin in CPT-treated/untreated samples (Fig. 5g). Cumulatively, these results clearly implied that ATM-mediated Vimentin activation during DNA damage responses is vital to exaggerate Vimentin function through halting of apoptotic progression and stimulate cellular migration.

\section{Inhibition of CPT-induced EMT by DIM confers anti- metastatic potential of DIM in vivo}

Next, the study with DIM in previous section encouraged us to further understand the potential of this drug, i.e. to increase the therapeutic efficacy of DNA damaging agent by abrogating the co-parallel EMT activation. In gelatin degradation assay, a significant abrogation of cellular invasion was noticed when HCT-116 cells were cotreated with CPT and DIM $(25 \mu \mathrm{M})$ compared to alone CPT-treated condition (Fig. 6a). Di indoyl methane is a potential antimetastatic drug reported to exhibit high bioavailability and low toxicity ${ }^{36}$; these properties of DIM led us to conduct a combinatorial study with CPT in Apc knock out murine model. The results clearly indicated a drastic alteration of crypt progenitor phenotype in CPT $(0.4 \mathrm{mg} / \mathrm{kg})$ in combination with DIM $(20 \mathrm{mg} / \mathrm{kg})$ as compared to CPT alone with $48 \mathrm{~h}$ (Fig. 6b). In this combination approach, both the expression of Vimentin and $\mathrm{p}^{\mathrm{ser} 38}$ Vimentin was significantly diminished but was otherwise substantially amplified in CPT-treated cohorts (Fig. 6c and Supplementary Fig. 9). Together, our results suggest a combinatorial therapeutic approach of Di indoyl methane with CPT enhanced the efficacy and potency of CPT by nullifying the associated EMT phenotypes.

\section{Discussion}

Majority of chemotherapeutic drugs induces DNA damage responses (DDR) signaling cascade leading to cell death. However, dysregulation of DDR pathways might result in hypersensitivity or resistance of tumors to therapy. Identification of the molecular mechanisms underlying this phenomenon is essential for enhancing the potential of cancer therapy ${ }^{37}$. The activation and stabilization of Snail-1 by ATM kinase and its further involvement in promoting metastasis poses vital questions concerning the simultaneous progression of apoptosis and EMT in cancer cells ${ }^{34,38}$. Many other groups also reported links between DDR and premature activation of pro 


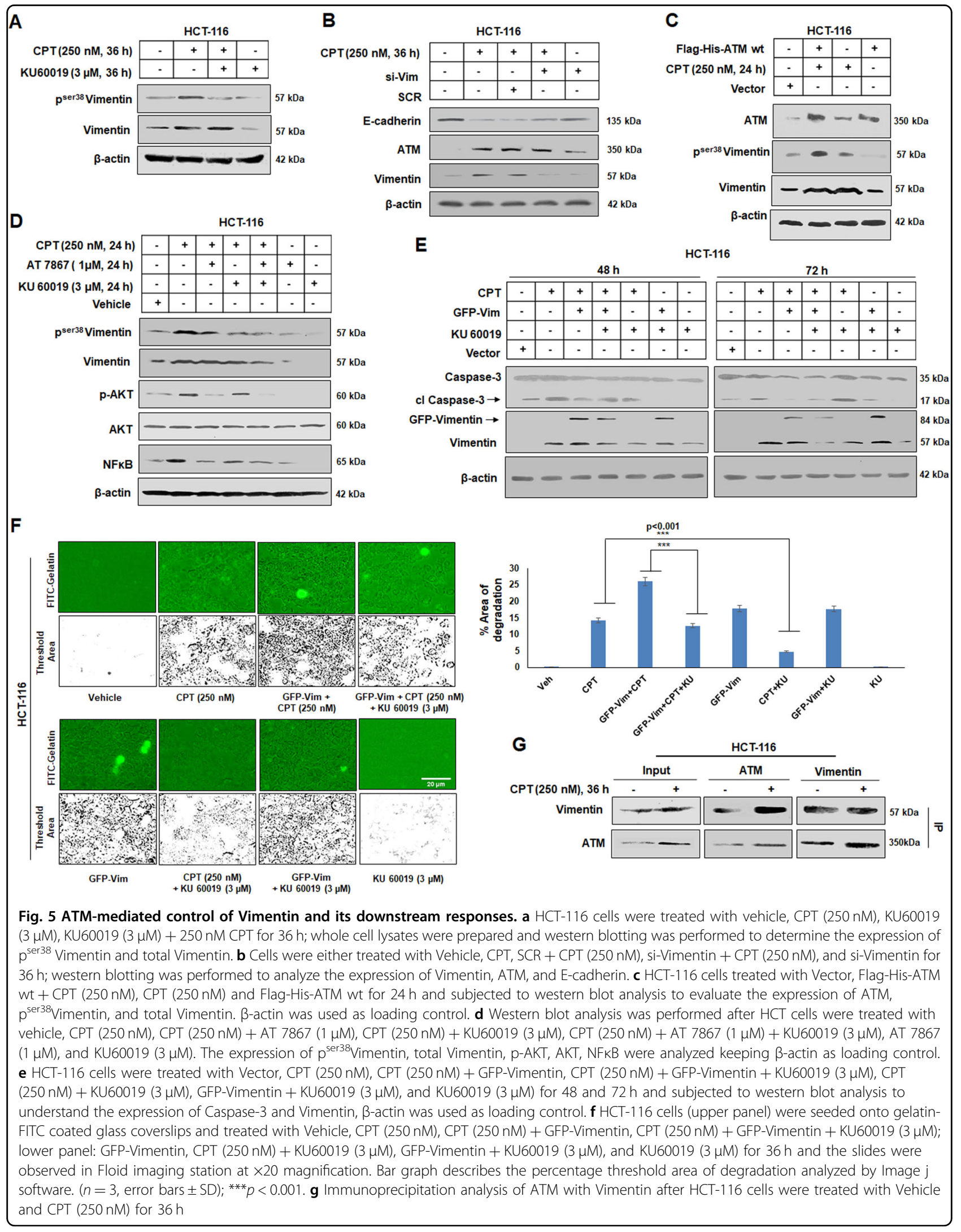




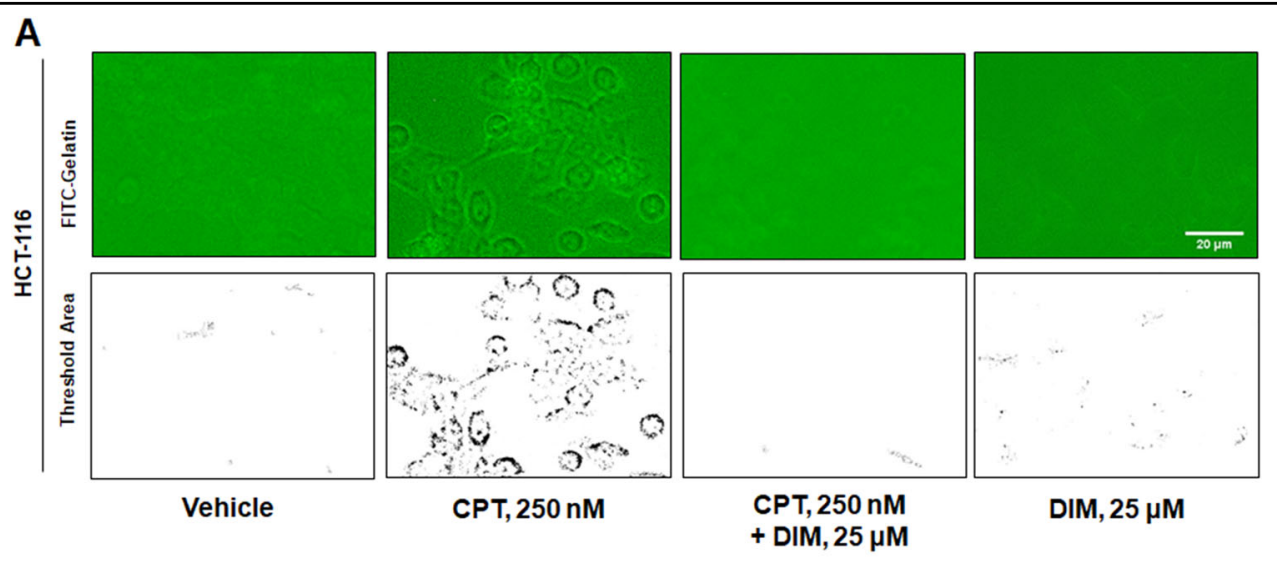

B
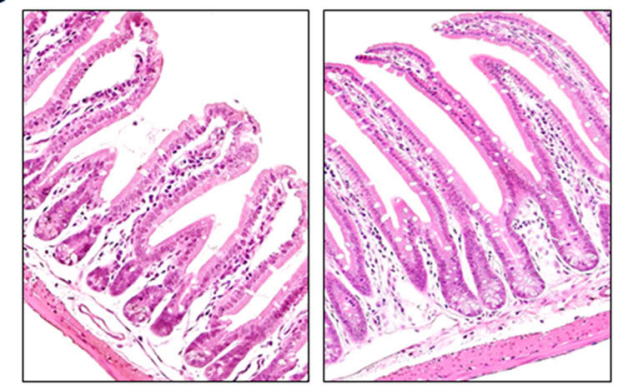

CPT, $0.4 \mathrm{mg} / \mathrm{kg}$
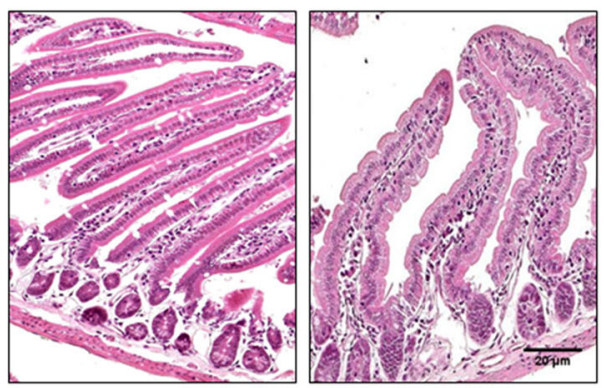

Vehicle

C

CPT, $0.4 \mathrm{mg} / \mathrm{kg}$
+ DIM, 20mg $/ \mathrm{kg}$

DIM, 20mg/kg
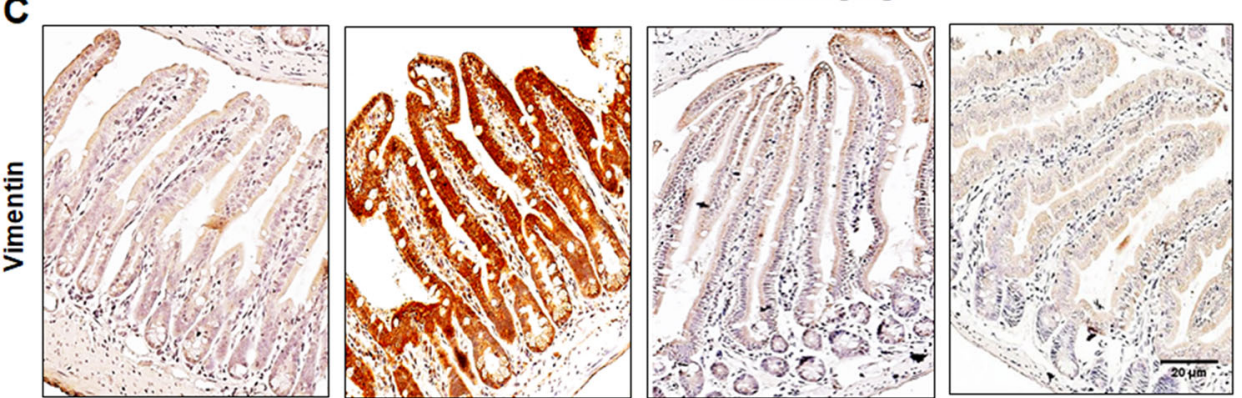

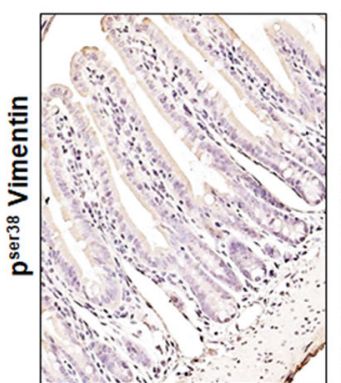

Vehicle
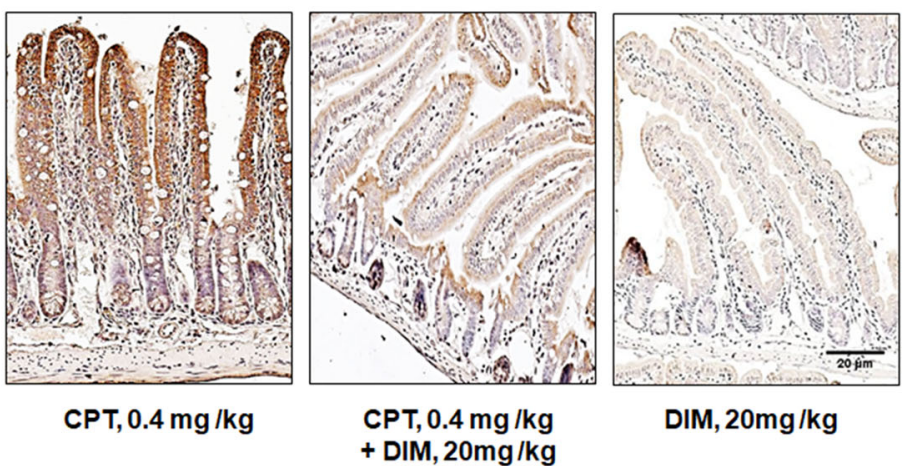

DIM, 20mg/kg

Fig. 6 DIM mediated abrogation of CPT-induced carcinogenesis and EMT. a HCT-116 cells seeded onto FITC-gelatin coated coverslips were treated with Vehicle, $250 \mathrm{nM}$ CPT, $250 \mathrm{nM}$ CPT $+25 \mu \mathrm{M}$ DIM, and $25 \mu \mathrm{M}$ DIM for $36 \mathrm{~h}$ and the slides were observed in fluorescence microscope at $\times 20$ magnification. $\mathbf{b}$ Hematoxylin and Eosin staining of intestinal tissue obtained from induced AhCre-ErT Apcfl/fl treated with Vehicle, $0.4 \mathrm{mg} / \mathrm{kg}$ CPT, $0.4 \mathrm{mg} / \mathrm{kg}+20 \mathrm{mg} / \mathrm{kg}$ DIM, and $20 \mathrm{mg} / \mathrm{kg}$ DIM for $48 \mathrm{~h}$. Images were taken at $\times 20$ magnification. c Immunohistochemistry analysis of Vimentin and $\mathrm{p}^{\text {ser38 }}$ Vimentin was performed on intestinal tissues obtained from AhCre-ErT Apcfl/fl mice treated with Vehicle, $0.4 \mathrm{mg} / \mathrm{kg}$ CPT, $0.4 \mathrm{mg} / \mathrm{kg}+20 \mathrm{mg} / \mathrm{kg}$ DIM, and $20 \mathrm{mg} / \mathrm{kg}$ DIM for $48 \mathrm{~h}$. Images were acquired in $\times 20$ magnification 


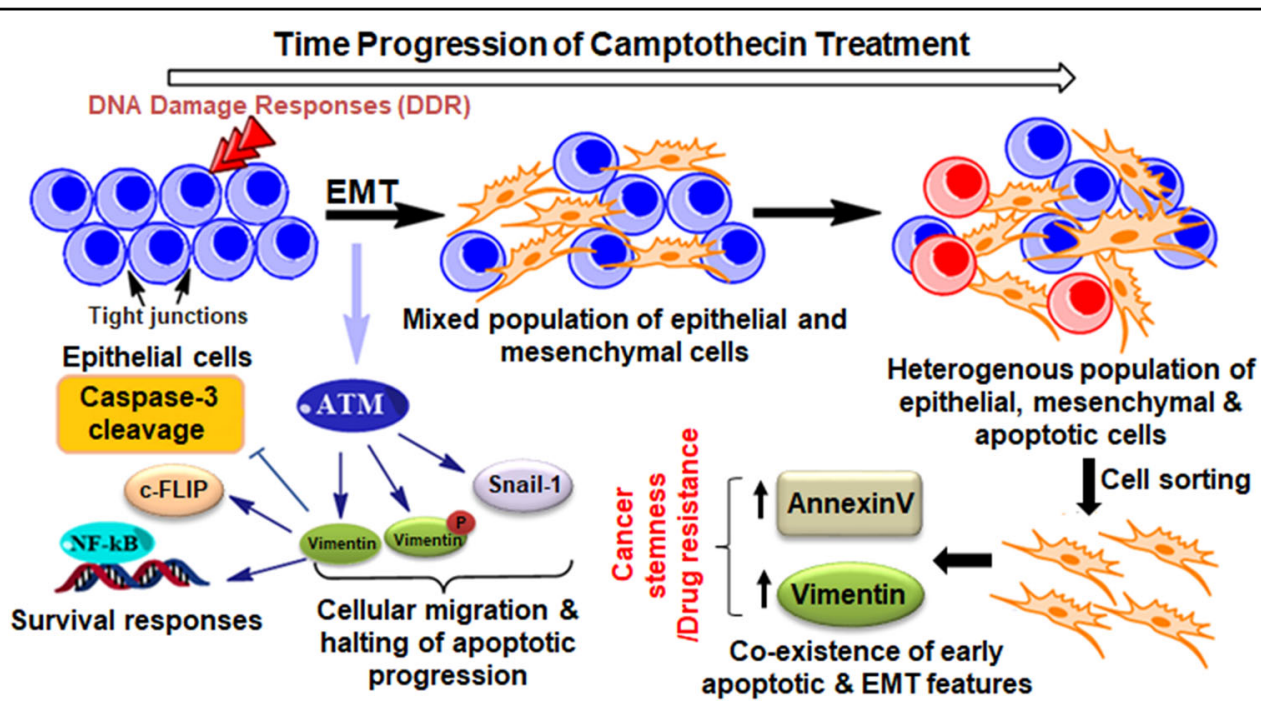

Fig. 7 Schematic representation of the proposed mechanism of action of the study depicting the co-existence of early-apoptotic and EMT features with time progression of Camptothecin treatment

metastatic and pro survival responses ${ }^{39,40}$ but evidences are not concrete. Incitement of EMT, indeed poses cellular survivability, but the activation of apoptotic machinery by CPT can't be overlooked ${ }^{14}$. In the present study we provide evidences that $\mathrm{CPT}$ treatment from $0-36 \mathrm{~h}$ activates signatures of EMT and cellular survival along with early apoptotic factors in cancer population both in vitro (HCT116, SW620, A549 cells) and in vivo (Apc floxed colorectal carcinoma model) conditions. But as the time progressed to $48 \mathrm{~h}$ the survival factors got attenuated and Caspase-3/ PARP-1 cleavage became more profound.

Both EMT and apoptosis were thought to be mutually exclusive fates involving distant machinery in a cell, until recently Ham et al. and David et al. clearly drew a connection between these two processes ${ }^{41,42}$. To shed light on this emerging concept of unification of these two evolutionarily conserved processes we found the existence of mesenchymal marker (Vimentin) in early apoptotic fraction of HCT-116 cells and inversely we have shown the binding of AnnexinV-mVenus to mesenchymal cells exhibiting migratory potential. Expression of Vimentin solely in $12 \mathrm{~h}$ time point within the viable population is undoubtedly a unique feature and currently our laboratory is envisaging on this interesting finding. Next step was to understand the mutual correlation of these two evolutionary significant biological processes-apoptosis and EMT, for that we employ Di-indole methane, a potential anti-metastatic molecule to nullify the associated EMT and we also silenced Vimentin by using si-RNA. In both the cases we have shown a shift in committed stages of apoptosis towards earlier time points and mitigation of pro-survival and pro-metastatic responses. The results also describe a positive signaling link between Vimentin and NFKB, which was feebly reported earlier by Huang et $\mathrm{a}^{43}$. further fortifying the less understood survival role of Vimentin apart from its well-studied structural functions (Fig. 7).

ATM kinase is a major DNA damage sensor and upon activation it halts the cell cycle and stimulate effectors of DNA repair pathways ${ }^{44}$. Additionally, activation of ATM kinase triggers several cellular survival responses and EMT which can be detrimental for the patients undergoing chemotherapy leading to further drug resistance ${ }^{45,46}$. Therefore, this research elucidates a novel signaling link between ATM kinase and Vimentin as we have shown that both these proteins physically interacts with each other and activated ATM kinase aids in maintain Vimentin in its phosphorylated form in ser38 region. Moreover, the results demonstrate that ATM plays a pivotal role in facilitating Vimentin to execute its functions related to hindering apoptosis as well as boosting invasion.

Finally, the anomalous activation of EMT and cellular survival pathways during initial phases of drug treatment challenges the basic structure of chemotherapy by DNA damaging agents and there is a grave need of designing new therapeutic approaches to prevent these collateral processes so that the tumor cells achieve committed stages of apoptosis efficiently resulting in successful therapeutics eliminating the problem of drug resistance.

\section{Materials and methods \\ Cell culture and reagents}

HCT-116 (colon carcinoma) and A549 (lung carcinoma) were purchased from American Type Culture Collection (ATCC, Manassas, VA, USA). HCT-116 and A549 cells were maintained in RPMI-1640 medium 
supplemented with $10 \%$ fetal bovine serum (Gibco, Carlsbad, CA, USA). HEPES, penicillin, streptomycin, sodium pyruvate and sodium bicarbonate were purchased from Sigma-Aldrich, St Louis, Missouri, US. The cells were maintained in a humified $\mathrm{CO}_{2}$ incubator (New Brunswick, Galaxy 170R) at $37{ }^{\circ} \mathrm{C}$ with $5 \% \mathrm{CO}_{2}$. CPT and $5 \mathrm{FU}$ were purchased from Merck, Kenilworth, NJ, U.S.A. and inhibitors z-VAD-FMK and KU60019 were obtained from Santa Cruz Biotechnology Inc (Santa Cruz, CA) and Merck (NJ, U.S.A) respectively. Di indoyl Methane (DIM) was purchased from TCI chemicals (Tokyo, Japan).

\section{Immunoblot analysis}

Immunoblot analysis was performed according to the standard procedure described previously by our group ${ }^{47}$. The list of antibodies and their dilutions are provided in Supplementary Table S1.

\section{Immunocytochemistry}

Immunocytochemistry experiments were carried out according to the established protocol of our laboratory ${ }^{48}$. The list of antibodies and their dilutions are provided in Supplementary Table S1. Images were taken using confocal microscope-Leica TCS SP8 (Leica Microsystem, Mannheim, Germany).

\section{In situ fluorescent gelatin degradation assay}

The matrix gelatin degradation assay was performed to assess the formation of invadopodia or podosomes. Following treatment, cells were analyzed by fluorescence microscope (Floid Cell Imaging Station) according to the method previously described ${ }^{48}$.

\section{Detection of mitochondrial membrane potential}

Mitochondrial membrane potential was analyzed by FACS (BD FACS CALIBUR, New Jersey, USA). $1 \times 10^{6}$ HCT-116 cells were seeded in $60 \mathrm{~mm}$ petri dishes and after overnight incubation the cells were treated with CPT for indicated time points. Upon termination the cells were trypsinized and $10^{6}$ cells $/ \mathrm{ml}$ suspension was prepared by serum free media, $400 \mathrm{nM}$ of TMRE dye was added and incubated for $20 \mathrm{~min}$ at $37^{\circ} \mathrm{C}$. After incubation cells were washed with PBS and resuspended in it, subsequently the TMRE fluorescence was measured by flow cytometer at excitation $/$ emission $=459 / 575$.

\section{AnnexinV-FITC/PI analysis}

HCT- 116 cells were seeded at $5 \times 10^{5}$ cells/well in $35 \mathrm{~mm}$ dishes and incubated for overnight at $37^{\circ} \mathrm{C}$ and $5 \% \mathrm{CO}_{2}$. Next day, the cells were treated with vehicle and CPT $(250 \mathrm{nM})$ for indicated time points. The AnnexinV-FITC experiments were performed using the AnnexinV-FITC Apoptosis Detection Kit according to the manufacturer's manual (Sigma-Aldrich, St Louis, Missouri, US). Briefly, cell pellets were re-suspended in $600 \mu \mathrm{L}$ binding buffer $(10 \mathrm{mM}$ HEPES [N-2- hydroxyethylpiperazine-N-2-ethane sulfonic acid], $140 \mathrm{mM} \mathrm{NaCl}$, and $2.5 \mathrm{mM} \mathrm{CaCl}_{2}, \mathrm{pH} 7.4$ ), and stained with $6.0 \mu \mathrm{l}$ Annexin V-FITC and $10 \mu \mathrm{l}$ propidium iodide staining solution for $20 \mathrm{~min}$ at RT in dark. The samples were successively analyzed by FACS (BD FACS Aria II) using BD Diva software.

\section{Cell sorting of AnnexinV-FITC positive cells}

Post staining with AnnexinV-FITC (as described in the above section), sorting of HCT-116 cells was performed in MoFlo cell sorter (MoFlo-Astrios EQ, Beckham-Coulter, Indianapolis, IN, US) as per the manufacturer's instruction. Further, the sorted cells were collected in HBSS (Hank's Balanced Salt Solution) from the sorter; then thoroughly rinsed with PBS and subsequently suspended in RIPA buffer for total protein extraction followed by western blotting.

\section{Sub cloning of sec AnnexinV-mVenus into human pEGFP-N3 vector}

Specific primers SecA5mV(Fw) and SecA5mV(Rev) (Integrated DNA Technology, lowa, USA), were used to obtain a $1.7 \mathrm{~kb}$ amplicon carrying AnnexinV-mVenus fusion protein with a secretary tag at $5^{\prime}$ end, from the template vector pTol2pA2-3.5ubb:secHsa.AnnexinVmVenus,crya:mCherry (gifted by Tjakko van Ham, Department of clinical genetics: Erasmus MC), in a PCR reaction with total volume of $50 \mu \mathrm{l}$ containing $5 \mu \mathrm{l}$ buffer (10×), $3 \mu \mathrm{MgSO}_{4}(25 \mathrm{mM}), 5 \mu \mathrm{l}$ dNTPs $(2 \mathrm{mM}), 5 \mu \mathrm{l}$ each primer $(3 \mu \mathrm{M}), 0.5 \mu \mathrm{l}$ of Vent DNA polymerase (New England BioLabs, Ipswich, Massachusetts, US), under the following conditions: $5 \mathrm{~min}$ at $95^{\circ} \mathrm{C}, 35$ cycles of $20 \mathrm{~s}$ at $95^{\circ} \mathrm{C}, 20 \mathrm{~s}$ at $50^{\circ} \mathrm{C}$ and $2 \mathrm{~min}$ at $75^{\circ} \mathrm{C}$. The amplicon and pEGFP-N3 target plasmid (Human expression vector) were double digested using EcoR1 and BamH1 along with Tango 10x buffer (Fermentas-Thermo Scientific) restriction enzymes. Digested products were gel eluted using Qia-quick gel extraction kit (Qiagen, The Netherlands) following manufacturer's protocol. The eluted product was ligated into the pre-digested pEGF-N3 plasmid using T4 DNA Ligase (Thermo Fisher Scientific, Waltham, Massachusetts, US) by incubating it at $37^{\circ} \mathrm{C}$ for $4-5 \mathrm{~h}$. The ligated product was further transformed in DH5 $\alpha$ competent cells. Transformed cells were spreaded on LB plates (with $50 \mu \mathrm{g} / \mathrm{ml}$ kanamycin) and incubated overnight at $37^{\circ} \mathrm{C}$ for further growth. The colonies were screened through colony PCR using same specific set of primers SecA5mV (Fw) and SecA5mV (Rev). Plasmids were isolated from positive colonies and were sequenced.

\section{Transient transfection with N3-secAnnexinV-mVenus construct}

$5 \times 10^{3}$ HCT-116 cells were seeded per well in an 8 well chamber slide and transfections were performed in Opti- 
MEM media (Thermo-Fisher Scientific, Waltham, Massachusetts, United States) using lipofectamine 3000 (Life Technologies, Carlsbad, California, USA) according to the manufacturer's instructions with some modifications. Subsequently, the cells were treated with CPT for indicated time points and observed under fluorescence microscope (Floid imaging station, Thermo Fisher Scientific, USA).

\section{Transient transfection with GFP-Vimentin construct \& pcDNA3.1(+) Flag-His-ATM wt}

GFP-Vimentin and pcDNA3.1(+) Flag-His-ATM wt constructs were kind gift from Howard Donninger (Assistant Professor, Department of Medicine, University of Louisville School of Medicine) \& Tej K. Pandita (Professor of Radiation Oncology, Houston Methodist Weill Cornell Medical College). $1 \times 10^{6}$ HCT-116 cells were seeded in $60 \mathrm{~mm}$ petri dishes and transfected with GFP-Vimentin and Flag-His-ATM wt constructs by using lipofectamine 3000. Transection efficiency was determined by both microscopy (for GFP-Vimentin) and western blotting.

\section{Transwell migration assay}

The cell invasion through Matrigel was carried out with the BD Biocoat Tumor Invasion Assay System (BD Biosciences, Bedford, MA) according to the manufacturer's instruction and as described previously ${ }^{49}$. To understand the co-existence of migration and apoptosis in HCT-116 cells, N3-secAnnexinV-mVenus transfected cells were seeded in transwell chambers (24 well format). Post CPT treatment $(36 \mathrm{~h})$, the cells were fixed in methanol and observed under a fluorescence microscope $(\times 20$ magnification).

\section{Cell scatter assay}

HCT-116 cells were seeded in a 6 well plate at a seeding density of 1000-2000 cells per well and kept for 4-5 days to develop small discrete colonies. Subsequently, the cells were transfected with N3-secAnnexinV-mVenus construct. Further, the transfectants were treated with $250 \mathrm{nM}$ CPT for $36 \mathrm{~h}$. After termination, the cells were washed with PBS and fixed with $4 \%$ paraformaldehyde, and then observed under fluorescence microscope $(\times 20$ magnification).

\section{D spheroid migration assay}

Microscopic spheroids of HCT- 116 cells were generated by hanging drop method ${ }^{50}$. Once visible spheroids were obtained, they were shifted to $1.5 \mathrm{~mm}$ Eppendorf tubes and centrifuged at 300-400 rpm for $5 \mathrm{~min}$. After washing the spheroids with PBS, they were embedded into Matrigel (BD MATRIGEL, BD Biosciences). Once the embedded mixture was solidified, RPMI media was added carefully. Subsequently, CPT treatment was given for indicated time points. The migration of cells from the core of the spheroids was observed under inverted microscope-20x magnification (Nikon Eclipse 200) and threshold images were created by using Image $j$ software (ver.1.50i, National Institute of Health, USA).

\section{Gelatin Zymography}

Gelatin Zymography was performed in order to understand the gelatinase activity of MMP-2 following a previously standardized method ${ }^{48}$.

\section{Cell cycle analysis by flow cytometry}

$0.5 \times 10^{6}$ HCT-116 cells were seeded in $35 \mathrm{~mm}$ petri dishes and treated with indicated concentration and time points of CPT and paclitaxel. After the treatment cells were processed for flowcytometry as described previously $^{51}$. Propidium iodide fluorescence was acquired by BD FACS Calibur.

\section{siRNA knockdown experiments}

siRNA targeting Vimentin was procured from SigmaAldrich (MISSION esiRNA, EHU151861) and transfection experiments were performed using lipofectamine 3000 (Life Technologies, Carlsberg, CA, USA) following manufacturer's instructions.

\section{Luciferase reporter assay}

To understand the effect of Vimentin signaling on $N F \kappa B$ expression, we employed NFKB promoter luciferase cassette (kind gift from Dr. Vivek M Rangnekar, University of Kentucky, Lexington, Kentucky) containing $\mathrm{NF \kappa B}$ responsive element obtained from $\mathrm{k}$ light chain enhancer placed upstream of SV-40 promoter in PGL2 (firefly luc) vector in two copies arranged in tandem ${ }^{52}$. $1 \times 10^{3}$ HCT-116 cells transfected previously with NFkBluc were seeded in a 96 well plate and transfection with siRNA for Vimentin was done. Subsequently, CPT treatment was given and the luciferase activity was measured with the help of a dual glow luciferase assay system (Promega, Madison, Wisconsin, United States) according to manufacturer's instructions.

\section{Co-immunoprecipitation assay}

Immunoprecipitation of both Vimentin and ATM kinase was performed following previously established method with minor modifications ${ }^{51}$. Briefly, $2.5 \times 10^{6}$ HCT-116 cells were seeded in $90 \mathrm{~mm}$ petri dishes and CPT/vehicle treatment was given for $36 \mathrm{~h}$. After the treatment, cells were harvested by gentle scrapping and washed with PBS; following centrifugation the pellets were suspended in RIPA buffer containing 1\% Triton X-100, 5 mM EDTA, $0.1 \%$ SDS, $1 \% \mathrm{NP} 4,1 \mathrm{mM}$ PMSF, $100 \mu \mathrm{M} \mathrm{Na}_{3} \mathrm{VO}_{4}$, and $1 \%$ 
protease inhibitor cocktail (Roche, Basel, Switzerland). Subsequently, the cell lysates were centrifuged at $14,000 \mathrm{rpm} / 10 \mathrm{~min}$ to remove the debris. Pre-clearing was performed with $25 \mu \mathrm{l}$ of Protein-G PLUS agarose beads (Santa-Cruz, Dallas, Texas, USA) and precleared lysates were further subjected to immunoprecipitation with $5 \mu \mathrm{g}$ antibody conjugated to $50 \mu \mathrm{l}$ of Protein-G PLUS agarose beads. The co-immunoprecipitated proteins were washed with RIPA buffer and further analyzed by western blotting.

\section{In vivo studies}

All animal studies were done in accordance with the protocols prescribed by the Institute Animal Ethical Committee of CCMB (IAEC), Hyderabad. The Apc knockout model (AhCre-ErT Apcfl/fl) is an inducible colon cancer murine model having crypt progenitor phenotype in the intestine upon induction with $\beta$-naphthoflavone and tamoxifen, leading to progressive knockout and death within 7 to 10 days' time. To analyze the effect of CPT-mediated DNA damage response to EMT in vivo, mice were divided into four different cohorts of three mice each including control group. Each cohort was further divided into two time points of 24 and $48 \mathrm{~h}$. For inducing $A p c$ knockout, intraperitoneal injections were given to 6 to 8 -week-old mice with $80 \mathrm{mg} / \mathrm{kg}$ $\beta$-naphthoflavone and tamoxifen (dissolved in corn oil at $10 \mathrm{mg} / \mathrm{ml}$ ) single dose per day till the end time point. Now respective cohorts were treated with Camptothecin, for that, oral gavaging of 10,20 and $30 \mu \mathrm{g}$ of CPT suspended in groundnut oil at $5 \mathrm{mg} / \mathrm{ml}$ was done, whereas control cohort received only groundnut oil. Thus, each mouse received $0.4,0.8,1.2 \mathrm{mg}$ of CPT as per $\mathrm{kg}$ of body weight. Dosage of the drug was decided by the fact that the curing dose of CPT starts from $1 \mathrm{mg} / \mathrm{kg}$. For combinatorial therapy, different cohorts of 3 mice each received $20 \mathrm{mg} /$ $\mathrm{kg}$ DIM, $20 \mathrm{mg} / \mathrm{kg}$ DIM in combination with $0.4 \mathrm{mg} / \mathrm{kg}$ $\mathrm{CPT}$ and $0.4 \mathrm{mg} / \mathrm{kg}$ CPT suspended in groundnut oil along with vehicle (groundnut oil) orally. Following treatment, animals were sacrificed as per the ethical guidelines at specific end time points followed by cardiac perfusion and tissues collection. The tissues harvested were preserved accordingly for all downstream experiments. Intestines were specifically cleaned in running tap water, opened up and fixed in methacam for $48 \mathrm{~h}$. The tissues were then made into a gut roll and preserved in $10 \%$ formalin for $48 \mathrm{~h}$ and after processing were sectioned at $4 \mu \mathrm{m}$ thickness.

\section{Immunohistochemistry}

Hematoxylin and Eosin (H \& E) and Immunohistochemistry were performed as follows. Sections were deparaffinized in xylene, rinsed in ethanol, and rehydrated. Heat induced epitope retrieval was performed by boiling slides in $10 \mathrm{mM}$ sodium citrate buffer $\mathrm{pH} 6.0$ for $20 \mathrm{~min}$ at $100^{\circ} \mathrm{C}$ and sections were cooled down at room temperature. Slides were then washed with $\mathrm{PBS}$ and blocked with $3 \% \mathrm{H}_{2} \mathrm{O}_{2}$ in methanol for 20 min to quench endogenous peroxidase activity. Subsequent washes of water and PBS were carried out and slides were blocked with $4 \%$ BSA in $1 \mathrm{X}$ PBS for $1 \mathrm{~h}$ at room temperature. Slides were then incubated with primary antibodies Vimentin, p-Vimentin, NF-kB (p65), all at 1:100 dilutions at $4{ }^{\circ} \mathrm{C}$ overnight. The next day slides were washed three times with PBST $(0.1 \%$ Triton-X) for $15 \mathrm{~min}$ each and incubated for two hours at room temperature with antirabbit HRP conjugated secondary IgG (Abcam ab97051). After incubation slides were again washed three times with PBST for 15 min each and liquid diaminobenzidine (DAB) (DAKO Envision) was used as a chromogenic agent for staining $(1 \mathrm{~min})$ and sections were counterstained with Mayer's haematoxylin. Negative controls of IHCs were prepared for each set by replacing primary antibody with PBS.

\section{Statistical analysis}

Data were represented as the mean \pm s.d of at least three independent experiments and $p$ value was calculated using GraphPad Prism software Version 5.0 (GraphPad Software, La Jolla, CA, USA). Student $t$ test were performed to compare the respective differences. $P \leq 0.05$ was consider as significant.

\section{Acknowledgements}

The work was supported by the institutional internal grant P-07 with institutional publication number IIIM/2296/2018. We thank our worthy Director Dr. R.A. Vishwakarma for encouraging us to accomplish this work. The authors are grateful to Council of Scientific and Industrial Research (CSIR) and Department of Biotechnology, Govt. of India, for providing fellowship to the research scholars.

\section{Author details}

${ }^{1}$ Academy of Scientific \& Innovative Research (AcSIR), CSIR-Indian Institute of Integrative Medicine, Jammu 180001, India. ${ }^{2}$ Cancer Pharmacology Division, CSIR-Indian Institute of Integrative Medicine, Jammu 180001, India. ${ }^{3}$ Cancer Biology, CSIR-Centre for Cellular \& Molecular Biology, Hyderabad 500007, India. ${ }^{4}$ Plant Biotechnology Division, CSIR-Indian Institute of Integrative Medicine, Jammu 180001, India. ${ }^{5}$ School of Biotechnology, Shri Mata Vaishno Devi University, Katra 182320, India

Conflict of interest

The authors declare that they have no conflict of interest.

\section{Publisher's note}

Springer Nature remains neutral with regard to jurisdictional claims in published maps and institutional affiliations.

Supplementary Information accompanies this paper at (https://doi.org/ 10.1038/s41419-019-1690-2).

Received: 25 December 2018 Revised: 24 April 2019 Accepted: 23 May 2019 Published online: 13 June 2019 


\section{References}

1. Kalluri, R. \& Weinberg, R. A. The basics of epithelial-mesenchymal transition. J. Clin. Investig. 119, 1420-1428 (2009).

2. Yilmaz, M. \& Christofori, G. EMT, the cytoskeleton, and cancer cell invasion. Cancer Metastasis Rev. 28, 15-33 (2009).

3. Satelli, A. \& Li, S. Vimentin in cancer and its potential as a molecular target for cancer therapy. Cell. Mol. Life Sci. 68, 3033-3046 (2011).

4. Arumugam, T. et al. Epithelial to mesenchymal transition contributes to drug resistance in pancreatic cancer. Cancer Res. 69, 5820-5828 (2009).

5. Shibue, T. \& Weinberg, R. A. EMT, CSCS, and drug resistance: the mechanistic link and clinical implications. Nat. Rev. Clin. Oncol. 14, 611 (2017).

6. Cheung-Ong, K., Giaever, G. \& Nislow, C. DNA-damaging agents in cancer chemotherapy: serendipity and chemical biology. Chem. Biol. 20, 648-659 (2013).

7. Gavande, N. S. et al. DNA repair targeted therapy: the past or future of cancer treatment? Pharmacol. Ther. 160, 65-83 (2016).

8. Blagosklonny, M. V. Analysis of FDA approved anticancer drugs reveals the future of cancer therapy. Cell Cycle 3, 1033-1040 (2004).

9. Hammond, W. A., Swaika, A. \& Mody, K. Pharmacologic resistance in colorectal cancer: a review. Ther. Adv. Med. Oncol. 8, 57-84 (2016).

10. Du, B. \& Shim, J. S. Targeting epithelial-mesenchymal transition (EMT) to overcome drug resistance in cancer. Molecules 21, 965 (2016).

11. Zhang, W. et al. Chemoresistance to 5-fluorouracil induces epithelial-mesenchymal transition via up-regulation of Snail in MCF7 human breast cancer cells. Biochem. Biophys. Res. Commun. 417, 679-685 (2012).

12. Parchment, R. E. \& Pessina, A. Topoisomerase I inhibitors and drug resistance. Cytotechnology 27, 149-164 (1998).

13. Beretta, G. L., Gatti, L., Perego, P. \& Zaffaroni, N. Camptothecin resistance in cancer: insights into the molecular mechanisms of a DNA-damaging drug. Curr. Med. Chem. 20, 1541-1565 (2013).

14. Han, Z. et al. Role of p21 in apoptosis and senescence of human colon cancer cells treated with camptothecin. J. Biol. Chem. 277, 17154-17160 (2002).

15. Luo, W., Fang, W., Li, S. \& Yao, K. Aberrant expression of nuclear vimentin and related epithelial-mesenchymal transition markers in nasopharyngeal carcinoma. Int. J. Cancer 131, 1863-1873 (2012).

16. Sansom, O. J. et al. Loss of Apc in vivo immediately perturbs Wnt signaling, differentiation, and migration. Genes Dev. 18, 1385-1390 (2004).

17. Tangudu, N. K. et al. RNA Interference Using c-Myc-Conjugated Nanoparticles Suppresses Breast and Colorectal Cancer Models. Mol. Cancer Therapeutics 14, 1259-1269 (2015).

18. Smith, B. \& Bhowmick, N. Role of EMT in metastasis and therapy resistance. J. Clin. Med. 5, 17 (2016).

19. Denecker, G. et al. Phosphatidyl serine exposure during apoptosis precedes release of cytochrome $\mathrm{c}$ and decrease in mitochondrial transmembrane potential. FEBS Lett. 465, 47-52 (2000).

20. Geske, F., Lieberman, R., Strange, R. \& Gerschenson, L. Early stages of p53induced apoptosis are reversible. Cell Death Differ. 8, 182 (2001).

21. Brauchle, E., Thude, S., Brucker, S. Y. \& Schenke-Layland, K. Cell death stages in single apoptotic and necrotic cells monitored by Raman microspectroscopy. Sci. Rep. 4, 4698 (2014).

22. Elmore, S. Apoptosis: a review of programmed cell death. Toxicol. Pathol. 35, 495-516 (2007).

23. Mohammad, R. M. et al. Semin. Cancer Biol. 35, S78-S103.

24. Morsch, M. et al. In vivo characterization of microglial engulfment of dying neurons in the zebrafish spinal cord. Front. Cell Neurosci. 9, 321 (2015).

25. Tiwari, N., Gheldof, A., Tatari, M. \& Christofori, G. Semin. Cancer Biol. 22, 194-207.

26. Vega, S. et al. Snail blocks the cell cycle and confers resistance to cell death. Genes Dev. 18, 1131-1143 (2004).

27. Huber, M. A. et al. NF-kB is essential for epithelial-mesenchymal transition and metastasis in a model of breast cancer progression. J. Clin. Investig. 114 569-581 (2004).

28. Piva, R., Belardo, G. \& Santoro, M. G. NF-kB: a stress-regulated switch for cell survival. Antioxid. Redox. Signal. 8, 478-486 (2006).
29. Matus, D. Q. et al. Invasive cell fate requires G1 cell-cycle arrest and histone deacetylase-mediated changes in gene expression. Dev. Cell 35, 162-174 (2015).

30. Nayak, D. et al. A therapeutically relevant, 3, 3'-diindolylmethane derivative NGD16 attenuates angiogenesis by targeting glucose regulated protein, 78 kDa (GRP78). Chem. -Biol. Interact. 232, 58-67 (2015).

31. Lee, G.-A., Hwang, K.-A. \& Choi, K.-C. Inhibitory effects of 3, 3'-diindolylmethane on epithelial-mesenchymal transition induced by endocrine disrupting chemicals in cellular and xenograft mouse models of breast cancer. Food Chem. Toxicol. 109, 284-295 (2017).

32. Burikhanov, R. et al. Arylquins target vimentin to trigger Par-4 secretion for tumor cell apoptosis. Nat. Chem. Biol. 10, 924 (2014).

33. Zhu, Q.-S. et al. Vimentin is a novel AKT1 target mediating motility and invasion. Oncogene 30, 457 (2011).

34. Sun, M. et al. Activation of the ATM-Snail pathway promotes breast cancer metastasis. J. Mol. Cell Biol. 4, 304-315 (2012).

35. Zhang, P. et al. ATM-mediated stabilization of ZEB1 promotes DNA damage response and radioresistance through CHK1. Nat. Cell Biol. 16, 864 (2014).

36. Reed, G. A. et al. Single-dose pharmacokinetics and tolerability of absorptionenhanced 3, 3'-diindolylmethane in healthy subjects. Cancer Epidemiol. Prevention Biomarkers 17, 2619-2624 (2008).

37. Goldstein, M. \& Kastan, M. B. The DNA damage response: implications for tumor responses to radiation and chemotherapy. Annu. Rev. Med. 66, 129-143 (2015).

38. Sun, M., Song, L., Zhou, T., Gillespie, G. Y. \& Jope, R. S. The role of DDX3 in regulating Snail. Biochim. Biophys. Acta Mol. Cell Res. 1813, 438-447 (2011).

39. Aller, P. et al. Camptothecin induces differentiation and stimulates the expression of differentiation-related genes in U-937 human promonocytic leukemia cells. Cancer Res. 52, 1245-1251 (1992).

40. Hudson, L. G. et al. Ultraviolet radiation stimulates expression of Snail family transcription factors in keratinocytes. Mol. Carcinog. 46, 257-268 (2007).

41. van Ham, T. J., Kokel, D. \& Peterson, R. T. Apoptotic cells are cleared by directional migration and elmo1-dependent macrophage engulfment. Curr. Biol. 22, 830-836 (2012)

42. David, C. J. et al. TGF- $\beta$ tumor suppression through a lethal EMT. Cell 164 1015-1030 (2016)

43. Huang, S.H. et al. Vimentin, a novel NF-kB regulator, is required for meningitic Escherichia coli K1-induced pathogen invasion and PMN transmigration across the blood-brain barrier. PlOS ONE 11, e0162641 (2016).

44. Maréchal, A. \& Zou, L. DNA damage sensing by the ATM and ATR kinases. Cold Spring Harb. Perspect. Biol. 5, a012716 (2013).

45. Stagni, V. et al. Tug of war between survival and death: exploring ATM function in cancer. Int. J. Mol. Sci. 15, 5388-5409 (2014).

46. Eich, M., Roos, W. P., Nikolova, T. \& Kaina, B. Contribution of ATM and ATR to the resistance of glioblastoma and malignant melanoma cells to the methylating anticancer drug temozolomide. Mol. Cancer Ther. 12, 2529-2540 (2013).

47. Rah, B. et al. A novel MMP-2 inhibitor 3-azidowithaferin A (3-azidoWA) abrogates cancer cell invasion and angiogenesis by modulating extracellular Par-4. PloS ONE 7, e44039 (2012).

48. Amin, $\mathrm{H}$. et al. Par-4 dependent modulation of cellular $\beta$-catenin by medicinal plant natural product derivative 3-azido Withaferin A. Mol. Carcinog. 55, 864-881 (2016).

49. Rasool, R. U. et al. Differential regulation of NM23-H1 under hypoxic and serum starvation conditions in metastatic cancer cells and its implication in EMT. Eur. J. Cell Biol. 96, 164-171 (2017).

50. Berens, E. B., Holy, J. M., Riegel, A. T. \& Wellstein, A. A cancer cell spheroid assay to assess invasion in a 3D setting. J. Vis. Exp. (105), e53409 (2015).

51. Nayak, D. et al. Inhibition of Twist1-mediated invasion by Chk2 promotes premature senescence in p53-defective cancer cells. Cell Death Differ. 24, 1275 (2017).

52. Nalca, A., Qiu, S. G., El-Guendy, N., Krishnan, S. \& Rangnekar, V. M. Oncogenic Ras sensitizes cells to apoptosis by Par-4. J. Biol. Chem. 274, 29976-29983 (1999). 